\title{
The development of a personalized patient education tool for decision making for postmenopausal women with osteoporosis
}

\author{
M. Hiligsmann ${ }^{1}$ - G. Ronda ${ }^{2}$ - T. van der Weijden ${ }^{2}$ - A. Boonen ${ }^{3}$
}

Received: 25 August 2015 / Accepted: 1 March 2016/Published online: 5 April 2016

(C) The Author(s) 2016. This article is published with open access at Springerlink.com

\begin{abstract}
Summary A personalized patient education tool for decision making (PET) for postmenopausal women with osteoporosis was developed by means of a systematic development approach. A prototype was constructed and refined by involving various professionals and patients. Professionals and patients expressed a positive attitude towards the use of the PET.

Introduction The purpose was to systematically develop a paper-based personalized PET to assist postmenopausal women with osteoporosis in selecting a treatment in line with their personal values and preferences.

Methods The development of the PET was based on a systematic process including scope, design, development of a prototype, and alpha testing among professionals and patients by semi-structured interviews.

Results The design and development resulted in a four-page PET prototype together with a one-page fact sheet of the different drug options. The prototype PET provided the personal risk factors, the estimated individualized risk for a future
\end{abstract}

Electronic supplementary material The online version of this article (doi:10.1007/s00198-016-3555-1) contains supplementary material, which is available to authorized users.

M. Hiligsmann

m.hiligsmann@maastrichtuniversity.nl

1 Department of Health Services Research, School for Public Health and Primary Care (CAPHRI), Maastricht University, P.O. Box 616, 6200 MD Maastricht, The Netherlands

2 Department of Family Medicine, CAPHRI, Maastricht University, Maastricht, The Netherlands

3 Department of Internal Medicine, CAPHRI, Maastricht University, Maastricht, The Netherlands major osteoporotic fracture and potential reduction with drugs, and a summary of advantages and disadvantages whether or not to start drugs. The drug fact sheet presents five attributes of seven drugs in a tabular format. The alpha testing with professionals resulted in some adaptations, e.g., inclusion of the possibility to calculate fracture risk based on various individual risk scoring methods. Important results from the alpha testing with patients were differences in the fracture risk percentage which was seen as worthwhile to start drugs, the importance of an overview of side effects, and of the timing of the PET into the patient pathway. All women indicated that the PET could be helpful for their decision to select a treatment. Conclusion Physicians and patients expressed a positive attitude towards the use of the proposed PET. Further research would be needed to test the effects of the PET on feasibility in clinical workflow and on patient outcomes.

Keywords Decision aid $\cdot$ Osteoporosis $\cdot$ Patient $\cdot$ Patient educational tool $\cdot$ Shared decision making

\section{Introduction}

There is robust evidence that drugs reduce the risk of osteoporotic fracture in postmenopausal women with established osteoporosis [1-3]. However, treatment nonadherence limits the (cost)-effectiveness of osteoporosis treatment in postmenopausal osteoporosis women that have been prescribed preventive drugs appropriately [4]. Poor uptake rates or poor adherence to therapy may be due to unaddressed patient values and preferences [5-7].

A way to facilitate patient understanding and to elicit patient preferences is the use of a patient education tool for decision making (PET) (or patient decision aid (PDA)) during the consultation [5]. A PDA is an 
evidence-based information tool that describes the available treatment options and their salient attributes and that also includes a value elicitation method and thereby can facilitate shared decision making $[5,8,9]$. A PET refers to a tool that does not include a value elicitation method. PET/PDAs are designed to support patients to make decisions when there is more than one reasonable option and when patients' personal preferences are relevant and important given the tradeoff between pros and cons of a single option [10]. Shared decision makingclinicians and patients making decisions together using the best available evidence-is more likely if the decision support tools have been developed for use in face to face clinical encounters [11]. PET/PDAs have been found to significantly improve patients' knowledge about the available options and to reduce decisional conflict. There is a variable effect of PET/PDAs on treatment choices including the uptake of medication [9]. The effects of PET/PDAs on adherence with the chosen option are inconclusive although there are some indications that PET/PDAs may increase patients' adherence to the treatment option of first choice $[9,11,12]$. A PET was previously developed and tested for the decision to start or not an oral bisphosphonate therapy in at-risk women with osteoporosis [5]. The PET was shown to improve the quality of clinical decisions of bisphosphonate therapy and improved the proportion of patients with high adherence level [5]. In recent years, new medications have however been introduced to treat osteoporosis which requires the development of a PDA that incorporates all possible medications. In addition, the assessment of personalized estimation of the risk of future major fracture [13] has become available. Recently, we also conducted qualitative and quantitative studies to get insights into the preferences of patients towards medication attributes $[14,15]$.

The aim of the current study was therefore to develop a new personalized PET for postmenopausal women with osteoporosis for use in face to face clinical encounters that assists individual patients who are considering options for drug treatment to prevent fractures in selecting a treatment that is in line with their personal values and preferences. To make PETs acceptable for the main stakeholders (patients and clinicians) and feasible for use in practice, a systematic approach for development with strong input from both patients and clinicians is recommended [16]. In this paper, we report about the findings and conclusions in the different steps of the developmental process.

Fig. 1 Development model of our PET until alpha testing, based on Coulter et al. [12]

\section{Methods}

Elements of the patient decision aid development process as described by Coulter et al. were followed, until the beta testing phase [16]. The key elements are shown in Fig. 1.

\section{Scope}

The project group discussed the exact scope of the PET including the target group, the type of decisions addressed, and the setting in which the decision aid would be used.

\section{Design}

The International Patient Decision Aid Standards (IPDAS) Collaboration describes criteria and minimum standards to assess the quality of PDAs, i.e., the quality of the development process and shared decision making design elements $[17,18]$. The six "qualifying" criteria that a tool should meet to be considered a PDA were included. Five items were about information dimension (providing information about options in sufficient detail to make a specific decision), and one item about values dimension (clarifying and expressing values). The items from the information dimension are as follows: the tool describes the health condition or problem for which the decision is required, the tool explicitly states the decision that needs to be considered, the tool describes the options available for the decision, the tool describes the positive features of each option, and the tool describes the negative features of each option. The item from the values dimension is as follows: the tool describes what it is like to experience the consequences of the options (e.g., physical, psychological, social) [17]. As we did not incorporate a value elicitation method, our tool is better named as a PET.

The clinical content and the format of our PET were based on evidence-based European and Dutch guidelines for the diagnosis and management of osteoporosis, literature about the assessment of individual fracture risk, guidelines for patients decision aids and short decision support tools for patients, literature about shared decision making and effective risk communication, previous qualitative and quantitative work about patients' preferences for osteoporosis drug treatment, and existing osteoporosis PETs.

\section{Prototype development}

The prototype was developed in an iterative process by the project team based on the information gathered in the design

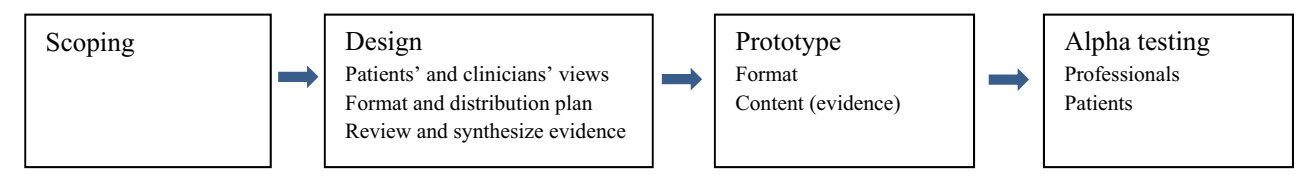


phase and previous studies including focus group [15] and a discrete-choice experiment [14].

\section{Alpha testing with professionals}

The prototype was refined in an iterative process involving various professionals including researchers, clinicians, and medical nurses. Eligible professionals were selected by the project team. Semi-structured face to face interviews were conducted to assess the acceptability and usability of the content and the format. We aimed for a minimum of five interviews. The interviews were audiotaped, and field notes were made during all the interviews. Afterwards, the interviews were listened to several times and compared and combined with the field notes. Quotations and comments of the patients were placed and summarized around the main elements of the prototype. The results from the interviews were discussed among the project group. Only one researcher (GH) performed the analyses, but this is a person without any conflict of interest in this field. It is an external researcher from an independent research group.

\section{Alpha testing with patients}

Semi-structured interviews were then conducted with outpatients to assess the comprehensibility and usability of the content and the format of the prototype. Eligible subjects (women with or at risk of osteoporosis) were recruited during their visit to the polyclinic by the physician or medical nurse. We aim to interview at least five subjects. The patients were confronted with the elements of the prototype PET and asked for their comments by asking questions and follow-up probes. The interviews were audiotaped, and field notes were made during interviews. The comments of the patients were summarized around the main elements of the prototype of the PET, using the method described above.

\section{Results}

\section{Scope}

The scope of the PET was to assist in clinical setting postmenopausal women in their decision to start anti-osteoporotic drugs in addition to life style changes and to help them in choosing the drug they preferred. There are two hierarchical steps to be distinguished within this decision: (1) Am I going to start drug treatment (Yes/No)? and if Yes, (2) Which drug do I prefer?

It was decided to develop a paper-based PET for use during the face to face clinical encounter in the polyclinic. Although web-based stand-alone decision aids are also developed that are meant to be used by patients independently in advance of the decision making consultation with the clinician, it was decided to develop a short paper-based PET as the clinicians feel the need to facilitate shared decision making with patients during the patient contacts. The advantage of using short decision support tools (minimal interventions) in clinical encounters is that they can facilitate patient involvement without substantially increasing consultation length $[5,19,20]$.

\section{Design phase}

\section{Patients' views}

The focus groups that were conducted to identify important osteoporosis medication attributes for patients showed that the most important attributes were effectiveness, side effects, and frequency and mode of administration [15]. The discretechoice experiment on treatment preferences for osteoporosis drugs revealed that patients prefer higher efficacy, lower costs, and less frequent dosing regimens. They preferred 6-month subcutaneous injection and oral monthly tablet, and disliked gastro-intestinal disorders more than other common side effects. It is noteworthy that preferences for treatment attributes varied significantly between patients [14], which justifies the development of a patient PET that facilitates individual decision making. In another study, it was found that the most common reasons expressed against bisphosphonate therapy were concerns about side effects or distrust of medications in general [7]. We included these findings in our PET.

\section{Literature review and consequences for the development of the PET}

The European and Dutch clinical practice recommendations provided guidance for identification of persons at risk and for management of postmenopausal osteoporosis. Recommendations for drug treatment are developed following GRADE guidelines [2, 21]. Although the fracture risk assessment tool (FRAX) is not standardly recommended for person with a recent fracture in the Netherlands [21], it might be assumed that patients will become more motivated to treatment adherence after being given a personal absolute risk score on developing a fracture in the next 10 years, by use of the FRAX-score, and that shared decision making about treatment will be facilitated. An estimated fracture risk is more important for patients than the more technical language of bone mineral density (T-scores). Literature suggests that patients appreciate graphical information about their risk of a certain disease and graphical information within a certain limited time frame is preferred above textual risk communication [22, 23]. Natural frequencies visualized by a population diagram appear to be an appropriate method to explain to patients their individual risk [23]. We therefore decided to include the FRAX-score for individual fracture risk assessment in our PET. 
After accounting additionally for current national recommendations and availability of reimbursement of specific drugs in the Netherlands, "alendronate" and "risedronate" were included as first choice drugs in our PET, and "denosumab," "zoledronic acid," "strontium ranelate," "raloxifene," and "ibandronate" as second choice drugs. Based on the overviews of the anti-fracture efficacy of these medicines for postmenopausal women with a high fracture risk, we communicated a mean risk reduction of $50 \%$ for future major osteoporotic fractures (clinical spine, forearm, hip, or shoulder fracture) independent of the specific drug.

\section{First prototype}

Two examples of decision aids in postmenopausal osteoporosis that match our purpose were found. For the decision whether or not to start drug treatment, the Osteoporosis Choice PET was developed at the Mayo Clinic in the US (http://shareddecisions.mayoclinic.org/decision-aids-forchronic-disease/other-decision-aids/) and conveys the pros and cons of treatment using an estimate of the patient's risk of bone fracture. For the second decision "Which drug do I prefer?," the so-called Option Grids (www.optiongrid.co.uk) matched with our purposes. An Option Grid is a one-page summary that provides answers to patients' frequently asked questions about specific attributes (characteristics) of the drugs organized in a tabular form. The specific features for each of the possible drug options are presented across the table columns [24]. As indicated above, in our case, the features or attributes of the available osteoporosis drugs (the options) were derived from previous qualitative and quantitative studies $[14,15]$. We used the above mentioned tools as well as the Dutch web-based stand-alone PET osteoporosis (http:// keuzehulpen.thuisarts.nl/keuzehulp-osteoporosebotontkalking-voorkomen-van-botbreuken), which was developed in parallel with the Dutch guidelines [21], for the development of our PET.

The results of the design phase resulted in the first prototype: a four-page PET together with a one-page fact sheet. The main elements of both are shown in Table 1.

\section{Alpha testing with professionals}

Two clinicians, two researchers, and two rheumatology specialist nurses were interviewed. The nurses indicated that the current consultation in the osteoporosis polyclinic is already extensive and comprehensive and that the PET should have proven added value to be acceptable in daily practice.

The most important comment concerning the content of the PET was about the fracture risk score and the time frame (within 3 years or 5 years instead of 10 years) that should be used to calculate the risk score of our target group adequately. Especially for patients with recurrent fractures and falls,
Table 1 Content of the first prototype of the PET including a one-page fact sheet

PET

Page 1

- What is osteoporosis?

- A patient's individualized fracture risk (\%) within the next 10 years based on the FRAX

Page 2

- An explanation of the natural course, the individualized risk percentage without drugs in natural frequencies and visualized by a population diagram

Page 3

- An explanation of the individualized risk percentage with drugs in natural frequencies and visualized by a population diagram

Page 4

-A summary of the choices (drug treatment or not) with their advantages and disadvantages

- Some words about the importance of a healthy lifestyle regardless of the choice

- Some considerations about individual preferences for certain drugs

Fact sheet

Presentation of seven drugs (options) that can help to prevent osteoporotic fractures with their attributes

- Which bones are predominantly protected?

- What is the mode of administration?

- What is the frequency of administration?

- How do you have to take the pill or powder or place of administration of injection or infusion?

- What are the side effects?*

*Instead of presenting side effects for each drug, the advice is to talk with the physician or medical nurse about the possible side effects of the various drugs

FRAX may underestimate the fracture risk. Two other models were mentioned by the clinicians and one researcher: the Garvan fracture risk calculator (GARVAN-FRC) (http:// www.garvan.org.au/bone-fracture-risk) and a nomogram developed by van Geel et al. [25]. They could be especially useful after a recent fracture.

One researcher had doubts about the applicability of a fact sheet in the Netherlands because it is meant in situations were shared decision making already takes place, and in the Netherlands, this might not yet be the case. This would imply that patients may be confronted with a fact sheet that presents a menu for anti-osteoporotic drugs that are in fact not on the menu of the prescribing clinician who is following his or her own routines or that of the team or institution where he or she is appointed.

\section{Second prototype}

As a result of these interviews, we included in the PET the possibility to calculate various risk scores with 10 -year and 5 - 
year risk prediction (FRAX, GARVAN-FRC, and the nomogram developed by Van Geel et al. [1]) dependent on the answers of the patients on the questions about risk factors, more specifically about recent fractures and falls. Moreover, we made a PET version with only a description of risk factors and the possibility to give an estimate of the fracture risk and the time frame as estimated by the clinician (the last prototype written in English is included in Appendix 1 (decision aid) and 2 (option grid)).

\section{Alpha testing with patients or persons at risk for osteoporosis}

Six females were interviewed, and the duration of each interview was between 12 and $28 \mathrm{~min}$. Five women were postmenopausal, aged 55 to 80 years, having either a prior fracture or a diagnosis of osteoporosis. Three women received osteoporotic drugs. We tested the content and format of the prototype mainly on comprehensibility and ability to help patients with decision making. We did not calculate a real individual fracture risk score but we used instead a fictitious number and a 10year timeframe.

The content and format of the four-page PET were discussed. First, subjects had to indicate whether they understood the percentages of the fracture risk and the risk reduction by visualizing the natural frequencies in a population diagram. All women indicated that the explanation of the risk rate illustrated by the "puppets" of the population diagram was clear for them.

"A graph or schematic diagram is always more clear than just a number or only narratively."

All women indicated that it could be helpful for their decision to take or not to take drugs. However, there were differences in the fracture risk percentage which was seen as worthwhile to start drugs. Women who have experience with (the consequences of fractures themselves or in their surrounding were more willing to take drugs, regardless of their fracture risk.

"My risk score should at least be $50 \%$."

"Only if my chance to break a bone becomes $0 \%$, I would take medicines, they harm your body always somewhere."

"A risk of $30 \%$ is well worth the effort to take medicines for me, considering my age, imagine that you break a bone. I have a friend who has broken a bone and I will do all that I can to prevent that."

"If you know the consequences of breaking a bone, you surely want a pill."
"I believe it is good to take medicines in my situation and I will see what happens."

"Side effects are the most important for me. So I first need to know the side effects."

Subsequently, the content and format of the fact sheet, presenting the attributes of the seven drugs (options), were discussed. The women indicated that the information was clear but that the format could be improved, e.g., the text size should increase to improve the readability.

The overview of the different drugs with their attributes was seen as an "eye opener," and women indicated that it surely could be helpful to choose a drug. They indicated that an overview of the most important or common side effects was important for them.

"Yes, but side effects are most important for me, so I first need to know the side effects. After that, I would choose for a drug that fits in my way of life, in my daily routine."

"Yes, it could be helpful. I don't like injections but eventually I would choose an injection because it is only 2 times a year."

"Yes, a tablet once a day you can easily forget, you have always to remember it, I would choose for a tablet once a month and mark it on the calendar (the first of each month). And I prefer a drug that is effective for all bones."

"Yes, it surely can help to choose a drug that fits to my daily life. I am not in a hurry in the morning but if you are (children and/or job), you probably will choose for an injection. A choice that fits better in your life can make it easier to continue."

"Yes, surely helpful to make a conscious choice. What effort you have to take for it, is an important issue. The various possibilities (and once a day, week, months) give you a better overview than just a tablet. Side effects are also important for me. I would like to know the most important ones, so that I know that if I have side effects, they might come from the drugs."

Finally, the introduction or timing of the fact sheet into the patient pathway maybe important for its usefulness, i.e., for some patients the first consultation in the polyclinic might be too early.

"To make a choice during this first consultation could be too much. I might already be surprised by the fact that I 
need drugs and by the technical terms. I think it might be better to present the options for drugs at a later time, e.g. at the family physician's office."

"I like the idea to have the possibility to switch my drug if it turns out that I have problems with the first one, that I have a choice, and that I can have an overview of the various possibilities. The table could therefore also be useful later on in the process."

\section{Discussion}

\section{Summary}

The development of our PET was based on a systematic development process that includes scoping, design, development of a prototype, and alpha testing with professionals and patients in an iterative process [16]. The content and format of the PET were based on a literature study including clinical guidelines, assessments of individual fracture risk, guidelines for PET/PDAs, shared decision making, risk communication, examples of other PETs, and studies about patients' preferences for osteoporosis drug treatment. Alpha testing with patients revealed that they all expressed a positive attitude towards the use of the PET.

This resulted in a four-page PET together with a one-page fact sheet. The PET provides the patient's individualized risk of having a major osteoporotic fracture based on absence or presence of risk factors (page 1), an explanation of the estimated risk (page 2), an explanation of the reduction of the fracture risk with drugs (page 3), advantages and disadvantages of the option "drugs" and the option "doing nothing," and some considerations about possible preferences for certain drugs (page 4). In the fact sheet, five attributes of seven drugs are presented in tabular form.

\section{Weak and strong points}

A potential limitation of our PET is that we did not develop all parts of PDAs that are prescribed by the IPDAS criteria as the tool will be integrated in a face to face consultation and is not to be used as stand-alone tool by the patient. The values dimension criteria - a PDA describes what it is like to experience the consequences of the options (e.g., physical, psychological, social) - is not fully elaborated in the tool itself, and this explains why we prefer named our tool as a PET. It is therefore important that during the consultation the clinician or the medical nurse stimulates the patient to make his own choice by thinking about the physical, psychological, and/or social consequences of the various options, for example: Can you imagine what breaking a bone means to you? Would it be a problem for you to follow the instructions for the intake of this specific drug?

An overview of the side effects of the drugs in the format of a summary in the fact sheet turned out to be more complicated than initially expected. Accurate interpretation of the risk of side effects is difficult because of various sources of information (e.g., randomized controlled trials versus case reports) that provide varying levels of evidence [26]. Moreover, the question was raised whether only the common side effects should be mentioned or also the serious but usually very rare side effects. The best way to communicate risk of side effects to patients would also need to be further investigated. This is an important issue for debate as the alpha testing with patients revealed that a summarized overview of the most common side effects is viewed as highly important to them. Therefore, it will be necessary to provide this information in the fact sheet. Experts on this field but also end users (patients) should be involved in such process. A recently updated review about the benefits and harms of pharmacologic osteoporosis treatments might also be helpful for that purpose although not all the drugs included in our fact sheet are included in the review [26]. Another potential weakness is that, although we recommend the patient to discuss non-pharmacological options (such as physical activity or nutrition) with doctors, our PET focused mainly on pharmacological options, and we therefore did not include an option grid for all possible nonpharmacological options. An integrated PET that would include both pharmacological and non-pharmacological options would be interesting.

Although the alpha testing phase yielded useful information for the further development of our PET, it should be mentioned that a relatively low number of professionals and patients were involved in this phase and that the patients were recruited from only one hospital. We can therefore not assure that the broad perspective of all potential users was captured. Before implementation of the tool at a large scale, it would be important to further test it in a larger and more representative group of patients with osteoporosis, the so-called beta testing of the tool. In addition, high-quality qualitative research (including assessment by two researchers) would be needed in further assessments of the tool and to investigate the most frequently asked questions and preferences of patients.

A close link between clinical practice guidelines and the content of PETs through collaborative development is important and can facilitate the implementation of PETs in clinical practice [16]. The clinical content of our PET was based on evidence-based Dutch and European guidelines, but a formal relation with the (Dutch) guideline shall be established when the tool will be tested in real practice. Another advantage of the current decision aid is the possibility to flexibly use different available risk score, improving the possibility to provide adequate individual risks. 


\section{Recommendations and considerations for further development, research, and implementation}

Besides the possible solutions for the weak points of the prototypes, i.e., stimulating the patient to make his or her own choice by helping the patient imagine what it is like to experience the effects of the options, and providing a summarized overview of the side effects, there are several other issues that need attention.

The discussion and research about the most appropriate fracture risk assessment tool, especially for patients with recurrent fractures and falls, is ongoing and may have consequences for the assessment of an individual risk score in our PET depending on the target group of the PET [25, 27, 28].

With regard to the choice of drugs by patients, there are differences between the Dutch and European guidelines $[2,21]$. The Dutch guidelines are more restricted with first- and second-line treatment options, and this may have consequences for the applicability of PET outside the Netherlands.

The lay-out of the fact sheet needs further improvement. Although a fact sheet is normally limited to one side of standard size paper and the information is purposefully limited [19], it will be a challenge to include the required information, including common side effects, in a more attractive and readable format.

The next step in the development process of the PET including the fact sheet is field or beta testing with patients and professionals in different settings. Field testing in "real world settings" is important to assess the feasibility of both tools, and feasibility is essential for implementation.

Many factors have been identified that might enable or prevent successful implementation of interventions in healthcare professional practice [29]. It is beyond the scope of this paper to discuss them all. Some factors important for future implementation came up in our study up to now. As mentioned before, a close link between guidelines and PETs is important and can facilitate the implementation of the PETs in clinical practice. The professionals who have to work with the tools need to be convinced of the added value of the PETs compared to their current procedures. Working with these tools has to fit in their daily routine or they have to be convinced that changing their daily routine would be efficacious. In a recent review, it was found that professional indifference, stemming from lack of confidence in the content of the PET/ PDAs and concern about disruption to established workflows, was an important barrier of implementation [30]. Another important issue that came up in our study is that patients need to be sensitive for the information at the moment of providing the information. Therefore, the timing of introduction of the tools into the patient pathway needs to be considered carefully.
Whether the use of the PET including the fact sheet will stimulate uptake rates or adherence rates for drug treatment is unknown. Montori et al. found a weak effect of an osteoporosis choice PET on medication adherence [5]. Research on the effects of PET/PDAs on medication uptake or treatment adherence concerning various conditions shows mixed or inconclusive results $[9,11,12]$. Our interviews with patients show that patient's thoughts about what risk and subsequently what fracture risk reduction is worth the effort to start drug treatment were quite diverging. In a study of patients' expectation of benefit from preventive drugs, it was found that the median value for the lower limit of benefit was $20 \%$ absolute risk reduction over 5 years [31]. This is an interesting outcome as it could mean that, if also applicable to our patients, even in our case with an assumed (relatively high) relative risk reduction of $50 \%$ with these drugs, for many patients, their expectation of benefit will be higher than the actual benefit provided by the drug. Possibly, the fact sheet, if fully applicable and presented at the right moment, may have the potential to increase adherence rates and to reduce discontinuation rates by offering patients the opportunity to choose a drug that fits to their daily life or to exchange one drug for another if it turns out that their first choice was not what they thought about it.

In conclusion, this study reports the development a PET to assist postmenopausal women with osteoporosis in selecting a treatment that is in line with their personal values and preferences. All patients interviewed during alpha testing expressed a positive attitude towards the use of a PET. Further development of the PET including the fact sheet, field testing, and research of their effects on patient outcomes will be next important steps.

Acknowledgments Financial support for this study was provided by Amgen (Zug, Switzerland). The funding agreement ensured the authors' independence in designing the study, interpreting the data, and writing and publishing the report. The authors have no conflict of interest to disclose. The authors thank all professionals and patients for their participation.

\section{Compliance with ethical standards}

Conflicts of interest Annelies Boonen received in the last five years research grants to her department form Pfizer, Abbvie, Merck, Amgen, and MSD, and occasionally speaker fees or advisory honoraria form Pfizer, Sandoz, and Abbvie. Mickael Hiligsmann has received research grants from Amgen, Servier, and SMB, Belgium. Gaby Ronda and Trudy van der Weijden declare that they have no conflict of interest.

Open Access This article is distributed under the terms of the Creative Commons Attribution-NonCommercial 4.0 International License (http://creativecommons.org/licenses/by-nc/4.0/), which permits any noncommercial use, distribution, and reproduction in any medium, provided you give appropriate credit to the original author(s) and the source, provide a link to the Creative Commons license, and indicate if changes were made. 


\section{References}

1. Crandall CJ, Newberry SJ, Diamant A, Lim YW, Gellad WF, Booth MJ et al (2014) Comparative effectiveness of pharmacologic treatments to prevent fractures: an updated systematic review. Ann Intern Med 161(10):711-723

2. Kanis JA, McCloskey EV, Johansson H, Cooper C, Rizzoli R, Reginster JY et al (2013) European guidance for the diagnosis and management of osteoporosis in postmenopausal women. Osteoporos Int 24(1):23-57

3. Reginster JY (2011) Antifracture efficacy of currently available therapies for postmenopausal osteoporosis. Drugs 71(1):65-78

4. Hiligsmann M, Rabenda V, Gathon HJ, Ethgen O, Reginster JY (2010) Potential clinical and economic impact of nonadherence with osteoporosis medications. Calcif Tissue Int 86(3):202-210

5. Montori VM, Shah ND, Pencille LJ, Branda ME, Van Houten HK, Swiglo BA et al (2011) Use of a decision aid to improve treatment decisions in osteoporosis: the osteoporosis choice randomized trial. Am J Med 124(6):549-556

6. Pencille LJ, Campbell ME, Van Houten HK, Shah ND, Mullan RJ, Swiglo BA et al (2009) Protocol for the Osteoporosis Choice trial. A pilot randomized trial of a decision aid in primary care practice. Trials 10:113

7. Scoville EA, Ponce de Leon Lovaton P, Shah ND, Pencille LJ, Montori VM (2011) Why do women reject bisphosphonates for osteoporosis? A videographic study. PLoS One 6(4):e18468

8. Giles K (2015) Decision aids for people facing health treatment or screening decisions. Int J Evid Based Healthcare 13(2):112-113

9. Stacey D, Legare F, Col NF, Bennett CL, Barry MJ, Eden KB et al (2014) Decision aids for people facing health treatment or screening decisions. Cochrane Database Syst Rev 1:CD001431

10. Stiggelbout AM, Van der Weijden T, De Wit MP, Frosch D, Legare F, Montori VM et al (2012) Shared decision making: really putting patients at the centre of healthcare. BMJ 344:e256

11. Joosten EA, DeFuentes-Merillas L, de Weert GH, Sensky T, van der Staak CP, de Jong CA (2008) Systematic review of the effects of shared decision-making on patient satisfaction, treatment adherence and health status. Psychother Psychosom 77(4):219-226

12. Stalmeier PF (2011) Adherence and decision AIDS: a model and a narrative review. Med Decis Mak Int J Soc Med Decis Mak 31(1): $121-129$

13. Kanis JA, Johnell O, Oden A, Johansson H, McCloskey E (2008) FRAX and the assessment of fracture probability in men and women from the UK. Osteoporos Int 19(4):385-397

14. Hiligsmann M, Dellaert BG, Dirksen CD, van der Weijden T, Goemaere S, Reginster JY et al (2014) Patients' preferences for osteoporosis drug treatment: a discrete-choice experiment. Arthritis Res Ther 16(1):R36

15. Hiligsmann M, van Durme C, Geusens P, Dellaert BG, Dirksen CD, van der Weijden T et al (2013) Nominal group technique to select attributes for discrete choice experiments: an example for drug treatment choice in osteoporosis. Patient Preference Adhes 7:133139

16. Coulter A, Stilwell D, Kryworuchko J, Mullen PD, Ng CJ, van der Weijden T (2013) A systematic development process for patient decision aids. BMC Med Inform Decis Mak 13(Suppl 2):S2

17. Joseph-Williams N, Newcombe R, Politi M, Durand MA, Sivell S, Stacey D et al (2013) Toward minimum standards for certifying patient decision aids: a modified Delphi consensus process. Med Decis Mak 34(6):699-710

18. Volk RJ, Llewellyn-Thomas H, Stacey D, Elwyn G (2013) Ten years of the International Patient Decision Aid Standards Collaboration: evolution of the core dimensions for assessing the quality of patient decision aids. BMC Med Inform Decis Mak 13(Suppl 2):S1

19. Elwyn G, Lloyd A, Joseph-Williams N, Cording E, Thomson R, Durand MA et al (2013) Option Grids: shared decision making made easier. Patient Educ Couns 90(2):207-212

20. Shepherd HL, Barratt A, Trevena LJ, McGeechan K, Carey K, Epstein RM et al (2011) Three questions that patients can ask to improve the quality of information physicians give about treatment options: a cross-over trial. Patient Educ Couns 84(3):379-385

21. Nederlandse Vereniging voor Reumatologie (2011) Richtlijn Osteoporose en Fractuurpreventie: derde herziening. Available from http://www.platformouderenzorg.nl/uploads/files/guidelines/ osteoporoseenfractuurpreventie.pdf

22. Tait AR, Voepel-Lewis T, Zikmund-Fisher BJ, Fagerlin A (2010) The effect of format on parents' understanding of the risks and benefits of clinical research: a comparison between text, tables, and graphics. J Health Commun 15(5):487-501

23. Waldron CA, van der Weijden T, Ludt S, Gallacher J, Elwyn G (2011) What are effective strategies to communicate cardiovascular risk information to patients? A systematic review. Patient Educ Couns 82(2):169-181

24. Bridges JFP, Hauber AB, Marshall D, Lloyd A, Prosser LA, Regier DA et al (2011) Conjoint analysis applications in health - a checklist: a report of the ISPOR good research practices for conjoint analysis task force. Value Health 14(4):403-413

25. van Geel TA, Nguyen ND, Geusens PP, Center JR, Nguyen TV, Dinant GJ et al (2011) Development of a simple prognostic nomogram for individualising 5-year and 10-year absolute risks of fracture: a population-based prospective study among postmenopausal women. Ann Rheum Dis 70(1):92-97

26. Rizzoli R, Reginster JY, Boonen S, Breart G, Diez-Perez A, Felsenberg D et al (2011) Adverse reactions and drug-drug interactions in the management of women with postmenopausal osteoporosis. Calcif Tissue Int 89(2):91-104

27. van den Bergh JP, van Geel TA, Lems WF, Geusens PP (2010) Assessment of individual fracture risk: FRAX and beyond. Curr Osteoporos Rep 8(3):131-137

28. van Geel TA, Eisman JA, Geusens PP, van den Bergh JP, Center JR, Dinant GJ (2014) The utility of absolute risk prediction using FRAX $(R)$ and Garvan Fracture Risk Calculator in daily practice. Maturitas 77(2):174-179

29. Flottorp SA, Oxman AD, Krause J, Musila NR, Wensing M, Godycki-Cwirko M et al (2013) A checklist for identifying determinants of practice: a systematic review and synthesis of frameworks and taxonomies of factors that prevent or enable improvements in healthcare professional practice. Implement Sci 8:35

30. Elwyn G, Scholl I, Tietbohl C, Mann M, Edwards AG, Clay C et al (2013) "Many miles to go ...": a systematic review of the implementation of patient decision support interventions into routine clinical practice. BMC Med Inform Decis Mak 13(Suppl 2):S14

31. Trewby PN, Reddy AV, Trewby CS, Ashton VJ, Brennan G, Inglis J (2002) Are preventive drugs preventive enough? A study of patients' expectation of benefit from preventive drugs. Clin Med 2(6):527-533 